\title{
Several Issues Should be Noticed in Corpora Selection of Pronouns Study in Excavated Documents of the Warring States

\author{
Yan-mei ZHANG ${ }^{1,2,}{ }^{*}$ and Han DING ${ }^{1}$ \\ ${ }^{1}$ School of Foreign Language, Guilin University of Electronic Technology, \\ Guilin, China \\ ${ }^{2}$ College of Liberal Arts, South China Normal University, Guangzhou, China \\ a576720717@qq.com, b34098482@qq.com
}

\begin{abstract}
Keywords: Excavated documents of the Warring States and Qin Dynasty, Pronouns study, Corpus selection.
\end{abstract}

\begin{abstract}
The passage points out that the Warring States era is a typical classical Chinese period so that it is worth studying pronouns in this period. Moreover, if the research uses excavated documents of the Warring States and utilizes new theoretical method, it will be of worth and significance. On this basis, the article discusses several issues which should be paid attention to of the corpus selection of the pronouns study of excavated materials of the Warring States from three aspects. Initially, the aspects are adding and absorbing new achievements in time and are taking as the research corpora. Secondly, though some excavated materials characters are few and plenty of it is hard to recognize, it should not be excluded from the research corpus. Finally, it should be treated with caution to excavated materials in different periods, regions and types when choosing corpora. It has enlightenment to taking up language studies of excavated materials.
\end{abstract}

\section{Introduction}

China, a country with an ancient civilization, is extremely abundant in ancient documents. The documents can be divided into handed-down documents and excavated materials.

Handed-down documents refer to documents that have spread after a long time before the discovery of underground documental materials. Taking the Warring States era as an example, Zuozhuan, Guoyu, Stratagems of the Warring States, The Analects of Confucius, Guan Zi, The Words of Mencius, Mo Zi, Lao Zi, Chuang-tzu, XunZi, Spring-Autumn of Lü, Han Feizi, The Songs of Chu, Yan Zi Chun Qiu are handed-down documents.

Excavated document is opposite to handed-down document. Once upon a century, plenty of ancient documental materials discovered from subsurface constantly. These documental materials underneath from underground are called excavated documents. Taking the Warring States era for example, excavated materials exceed fruitful, including bronze inscriptions in Qin Dynasty of the Warring States, scripts from bamboo slips in Qin Dynasty of the Warring States, silk books of the Warring States and jade text in Qin Dynasty of the Warring States. Among them, scripts from bamboo slips in Qin Dynasty of the Warring States include Xinyang bamboo slips of Chu, Wulipai bamboo slips of Chu, Angtian Lake bamboo slips of Chu, Yang Jia Wan bamboo slips of Chu, Wang Mountain bamboo slips of Chu, Jiu Dian bamboo slips of Chu, Bao Mountain bamboo slips of Chu, Guo Dian bamboo slips of Chu, Shanghai Museum bamboo slips of Chu, Xincai bamboo slips of Chu, bamboo slips in The 
Chinese University of Hong Kong, bamboo slips of Chu in Tsinghua University, Shuihudi bamboo slips of Qin, Qingchuan bamboo tablets of Qin, Fangma Beach bamboo slips of Qin, Yue Shan bamboo tablets of Qin, Longgang bamboo slips of Qin, Choujiatai bamboo slips of Qin, Liye bamboo slips of Qin, bamboo slips of Qin in Peking University and Tomb of Marquis Yi of Zeng bamboo slips. Jade text in Qin Dynasty of the Warring States include Yin jade marginal inscription of Qin, Xing Qi jade inscription, jade Huang admonitions carved on a stone, ShouQiulithoglyph, ten Qin kingdom inscribed steles, Mausoleum Tablet and the engravings on Mount Yi.

Excavated document has a strong authenticity of document because it has been hidden underground without spreading over a long period and veritably keeps its appearance at that time. It should be said that in the case of grammar study of ancient Chinese, excavated document corpus has greater scientific value than handed-down document that hard to keep its original language and may not have clear era and region.

The Warring States era is a typical classical Chinese period so that it is worth studying pronouns in this period. Researches would be worthy and significant when using the corpus of excavated materials of the Warring States and its new theoretical methods.

Then, what should we pay attention to when selecting corpus of pronouns studies of excavates materials of the Warring States?

\section{Issues Should be Paid Attention to When Selecting Corpus of Pronouns Studies of Excavates Documents of the Warring States}

\section{New Achievements Should be Added and Absorbed in Time and Taken as the Research Corpora}

For the time being, some excavates documents corpora of the Warring States have not been announced and some have been published but are hard to recognize when researching. Thus, there necessarily exists an issue of lag research materials used. Therefore, a subsequent researcher must take note of adding and absorbing new achievements in time and take them as the research corpora.

For instance, the passage called The Amount and Syntactic Functions of the First Person Pronoun 'wu' of The bamboo slips of Chu State in the Warring States Period collected by the Shanghai Museum --and Consult with Grammar Study of The bamboo slips of Chu State in the Warring States by Mingxiao Li ('The First Session Ancient Chinese Characters and Language Research of Excavated Documents International Symposium' Conference Collected Papers, December 2016)that mentioned:

Counting from Grammar Study of The bamboo slips of Chu State in the Warring States by Mingxiao Li, 'The bamboo slips of Chu State in the Warring States Period collected by the Shanghai Museum has altogether 89 examples of "wu", including 71 examples used as subjects, four examples as objects and 14 examples as attributes'.

However, our statistical results are 140 examples of 'wu' used as the first person pronoun in The bamboo slips of Chu State in the Warring States, including 100 examples used as subjects, four examples as objects (one example to be determined, preliminary decided as object), 31 examples as attributes, one example as a subject-object structure, one example as a complement and three examples to be determined.

The most important reason of causing the difference is the reason of corpora. Though Grammar Study of The bamboo slips of Chu State in the Warring States 
(Mingxiao Li, 2010) used many newly excavated document corpora at that time, the preface of the book clearly demonstrated that the Shanghai Museum bamboo slips corpora adopted then could only limit in The bamboo slips of Chu State in the Warring States Period collected by the Shanghai Museum (Part One to Seven):

Until now, the excavated bamboo slips of Chu have been more than twenty batches. But owing to a variety of reasons, some bamboo slips have not been established or only parts of them have been established. The book could only be directed against materials collected to do researches. The materials which are based by the book are 14 batches in total. Mainly are according to corruption explanations of the book to report and refer to the latest research achievements to complement, redefining corpora. Basic corpora are mainly Changsha Wulipai M406 bamboo slips, Changsha Angtian Lake M25 bamboo slips, XinyangChangtaikwan M1 bamboo slips, Jiangling Wang Mountain M1 and M2 bamboo slips, Suizhou Tomb of Marquis Yi of Zeng bamboo slips, Jiangling Jiudian Tomb of Chu bamboo slips, Mount Weld Sunset Slope M2 bamboo slips, Jingmen Bao Mountain M2 bamboo slips, Jiangling Brick and Tile Factory Number M370 bamboo slips of Chu, HuanggangCaojiagang bamboo slips of Chu, Jingmen Guo Dian bamboo slips of Chu, Xincai bamboo slips of Chu, bamboo slips of Chu state in the Warring States period collected by the Shanghai Museum (Part One to Seven) and bamboo slips of Chu in the heritage hall of The Chinese University of Hong Kong.

It has been over six years since Grammar Study of Bamboo Slips in the Chu Dynasty of the Warring States (Mingxiao Li, 2010) published. Now we are lucky to see such The bamboo slips of Chu State in the Warring States Period collected by the Shanghai Museum (Part Eight and Nine) mature corruption explanations and regard them as research corpora. Thus, as a later researcher, it is beyond doubt to add them in it.

\section{Though Some Excavated Materials are Few and Plenty of it is Hard to Recognize, it Should not be Excluded from the Research Corpus}

Excavated document materials, hard to tackle, owing to limitations of various conditions when studying, there are many confinements to aspects of mastering, using and comprehending ancient Chinese characters and excavated document materials occasionally. For instance, some are not completely explained and read while some materials were damaged, incomplete and words blurred when unearthed. But with regard to these materials, can we exclude them when researching? We consider that the answer to the question is no.

We take three lots bamboo slips of the Warring States unearthed in Changsha, Hunan, China (Wulipai bamboo slips of Chu, Angtian Lake bamboo slips of Chu and Yang Jia Wan bamboo slips of $\mathrm{Chu}$ ) as examples.

Wulipai bamboo slips of Chu, excavated from a batch of bamboo slips of the Warring States from the Chu tombs of the Warring States in No. 406, Wulipai, eastern suburbs of Changsha, Hunan in 1951. Excavated bamboo slips are 38 in total, every of them broken without a complete. By patchwork, 18 of them recovered. Its contents are the ancient records of funerary items in the funeral activities list, with upper section of the bamboo slip recording names and numbers of implements and lower section recording the position of implements. According to the corruption explanations and interpretations of these bamboo slips of Chu, it can be seen from $A$ Report on the Excavation of Changsha (published by Science Press, 1957) compiled by the Institute of Archaeology of Chinese Academy of Sciences and Bamboo Slips of Chu of the Warring States Collection written by Chengzuo Shang (Qilu Press, 1995). 
The former established photos of 21 bamboo slips as well as copies and part of interpretations of 38 bamboo slips, while the later published photos, copies and part of interpretations of 18 bamboo slips pieced together and recovered.

Angtian Lake bamboo slips of Chu, excavated from a batch of bamboo slips of the Warring States from the Chu tombs of the Warring States in No. 25, Angtian Lake, Changsha, Hunan in 1953. Excavated bamboo slips are 43 in total, in which 19 bamboo slips are complete. Its contents are also the ancient records of funerary items in the funeral activities list. About the corruption explanations and interpretations of these bamboo slips of Chu, it can be seen from Changsha Angtian Lake Bamboo Slips of Chu Research Document Headlines from XuerenXu (Bamboo Silk Research Website, June 1, 2003), which listed research monographs of these bamboo slips of $\mathrm{Chu}$ in details. For instance, research monographs are Researches on Changsha Angtian Lake Excavated Bamboo Slips of Chu written by Shuqing Shi (QunLian Press, June 1955), Bamboo Slips of Chu of the Warring States Collections (Qilu Press, 1995) and Chu Tombs of Changsha edited by Hunan Provincial Museum (Cultural Relics Publishing House, January 2000).

Yang Jia Wan bamboo slips of Chu, excavated from a batch of bamboo slips of the Warring States from the Chu tombs of the Warring States in No. 6, Yang Jia Wan, northern suburbs of Changsha, Hunan in 1954 (Chu Tombs of Changsha edited by Hunan Provincial Museum changed its number to M569). Excavated bamboo slips are 72 in total, in which every bamboo slip just had one or two characters and many of them are faded and practically indecipherable. Chien-Wenis different from general common the ancient records of funerary items in the funeral activities list, so educational circles think it should be further studied whether its characteristics is the ancient records of funerary items in the funeral activities list or not. About the corruption explanations and interpretations of these bamboo slips of Chu, it can be seen from Bamboo Slips of Chu of the Warring States Collection written by Chengzuo Shang (Qilu Press, 1995) and Chu Tombs of Changsha edited by Hunan Provincial Museum (Cultural Relics Publishing House, January 2000).

To be sure, it is three batches of bamboo slips of the Warring States excavated in Changsha from 1952 to 1954 (namely Wulipai bamboo slips of Chu, Yang Jia Wan bamboo slips of Chu and Angtian Lake bamboo slips of $\mathrm{Chu}$ ) that let people witnessed the real object of bamboo slips of Pre-Qin Dynasty for the first time. It definitely caused sensations of all circles at that time (the Institute of Archaeology of Chinese Academy of Sciences also found bamboo slips of the Warring States period in the northern suburb of Changsha in 1951, but the characters were hard to recognize). Among them, Angtian Lake bamboo slips of Chu have kept in a good condition and had a clear script. Number 25 Wooden-chambered Tombs of Angtian Lake in Changsha (Journal of Archaeology, second periodical in 1957) mentioned that it was Angtian Lake bamboo slips of Chu that excavated with clear scripts and many character numbers in bamboo slip brochures of the Warring States for the first time. Even so, as character numbers are relatively few and meanwhile, some characters are hard to recognize (especially the character numbers of Wulipai bamboo slips and Yang Jia Wan bamboo slips are quite few. In particular, 72 bamboo slips of Yang Jia Wan bamboo slips could be seen that characters of 50 bamboo slips but quite a lot of characters are indistinct. According to Fourteen Excavated Bamboo Slips Brochures of the Warring States in Chu Region written by Wei Chen, only eight characters could be recognized in 24 bamboo slips with inks embodied).

On the basis of characters able to be recognized and current corruption explanations and interpretations, we can see that only one example of pronoun 'qi' could be seen in 
Angtian Lake bamboo slips of Chu. Though there are two examples of 'qi' in Wulipai bamboo slips of Chu, according to Bamboo Slips of Chu of the Warring States Collection written by Chengzuo Shang (Qilu Press, 1995), 'qi' should be explained as an object name 'ji'(which means dustpan in Chinese). Thus, there is no pronoun in Wulipai bamboo slips of Chu (the annotation from page 29 of Grammar Study of The bamboo slips of Chu State in the Warring States by Mingxiao Li is wrong) while there is no pronoun of eight recognized characters in Yang Jia Wan bamboo slips of Chu.

Therefore, from the perspective of pronouns study of excavated documents of the Warring States, the materials are too far from enough to demonstrate the issue.

Despite this, we consider that such kind of materials with few words and many of them hard to be recognized should not be excluded from research corpora. One reason is that these materials belong to a family member of excavated documents of the Warring States. We should not exclude them for the reason that pronouns have few numbers and hard to be recognized, otherwise what we have studied could not be exhaustive and comprehensive. Another reason is that some characters hard to be recognized or explained to read have the possibility to be explained through research achievements of grammar in substance.

\section{It Should be Treated with Caution to Excavated Materials in Different Periods, Regions and Types when Choosing Corpora}

When choosing corpora, it is another very important point that we must be careful to treat various periods, regions and types of excavated documents.

Mr. Dekuan Huang, president of Chinese Character Association, discussed the form, copy, burial era and region of excavated materials as well as the determination of version being spread and transcribed situation in Issues of Researching and Using Excavated Document Data in History of Chinese Language ('The First Session Ancient Chinese Characters and Language Research of Excavated Documents International Symposium' Conference Collected Papers, December 2016). The essay makes a decisive significance to choosing and using research corpora of the history of Chinese language. It points out discrepancies of corpora values of excavated documents in different times and types, considering that not all 'excavated materials' equal to 'parallel information'. Also, the passage strengthens to attach importance to researches having characteristics and properties of excavated documents so as to avoid falling into 'trap of new materials' when using excavated documents corpora to study the history of Chinese language by giving examples and analyzing the differences between Guo Dian, silk manuscripts from Mawangdui, Peking University bamboo slips of Han Dynasty and $L a o Z i$ in four versions written by Wang Pi.

Mr. Yujin Zhang also discussed different types as well as times and regions issues of excavated documents materials of the Warring States. He mainly mentioned three points. Firstly, it should be noticed that different types of documents in the same period may occur to language characteristics of diverse era when choosing corpora. For instance, excavated documents of the Warring States can roughly be divided into two forms. One form is archives, namely documents and another form is classics, also known as ancient books. Excavated documents of archives, overall, the time of its form was close to the period of unearthed tombs(era lower bound of this kind of document) and some could even be synchronal, like documents of bamboo slips specially recorded burial articles. The form era of excavated documents of classics often fairly far away from its tomb period and some may even have long distances such as The Book of Changesof bamboo slips of Chu State collected by the Shanghai Museum. Though the documents excavated from tombs of the Warring States and Qin 
Dynasty, if its form era was earlier than the turn of the Spring and Autumn and Warring States, this kind of document should be excluded when researching excavated documents grammars of the Warring States. Secondly, the era of excavated materials documents should be noted. A kind of document can be modified regularly (sometimes exists errors) after its form and in the spreading process. Thus, excavated documents mirror the language appearance from its form era to tomb period (era lower bound). So according to a kind of document, we should not only know its tomb era but also possibly discuss its form era and circulation situation. Thirdly, the region of excavated documents materials should be noticed. Some document region issues are complicated as their excavated region and the region of writers do not agree. For example, Black Clothes in Guo Dian bamboo slips of Chu, unearthed from the Chu tombs, its author maybe a person from Lu. Hence, it is inappropriate to treat Guo Dian bamboo slips as documents in Chu region.

In short, when selecting corpora, we must be careful to treat different periods, regions and types of excavated documents. Only when choosing certain materials via carefully selecting and repeated identification and document materials that can objectively reflect the appearance of the era can we ensure scientific and reliable research result.

\section{Conclusion}

In conclusion, the Warring States era is a very important period in Chinese history. Its language is typical classical Chinese so that it is worth researching pronouns of this period. Excavated documents have advantages of keeping the original appearance of language and clear time and region so that the corpora can be used to study pronouns system of the Warring States era. It has great scientific significance to establish an ancient Chinese grammar and vocabulary history that conforms to the historical reality to a higher degree.

When utilizing a kind of precious corpus that excavated materials of the Warring States to study pronouns, we should notice that three aspects selecting corpora: Firstly, the aspects are adding and absorbing new achievements in time and are taking as the research corpora. Secondly, though some excavated materials arefew and plenty of it is hard to recognize, it should not be excluded from the research corpus. Finally, it should be treated with caution to excavated materials in different periods, regions and types when choosing corpora. It also means that in the pronouns study of excavated documents, we ought to note that excavated documents in various time and types vary in corpora value.

\section{Acknowledgement}

The article got advice from Mr. Yujin Zhang, the professor and doctoral supervisor of College of Liberal Arts, South China Normal University. It was also supported by the Project of National Social Science Foundation in 2013, with the project index number 13BYY106, in which the content was 'content words study of excavated documents of the Warring States and lexicography'. Thanks again.

\section{References}

[1] Dekuan Huang, Issues of Researching and Using Excavated Document Data in History of Chinese Language, 'The First Session Ancient Chinese Characters and 
Language Research of Excavated Documents International Symposium' Conference Collected Papers, December 2016.

[2] Yujin Zhang, A Research on Chinese Pronoun of the Western Zhou Dynasty, ZhongHua Book Company, Beijing, 2006.

[3] Yujin Zhang, A Research on Function Words in Excavated Materials of the Warring States, People press, Beijing,2011.

[4] Yanmei Zhang, The Amount and Syntactic Functions of the First Person Pronoun 'wu' of The bamboo slips of Chu State in the Warring States Period collected by the Shanghai Museum --and Consult with Grammar Study of The bamboo slips of Chu State in the Warring States by Mingxiao Li, 'The First Session Ancient Chinese Characters and Language Research of Excavated Documents International Symposium' Conference Collected Papers, December 2016.

[5] Yanmei Zhang, etal, The Retrospect and the Prospect of a Research on Pronouns in Excavated Materials of the Warring States, 2016 International Conference on Contemporary Education, Social Sciences and Humanities (ICCESSH 2016), St.Petersburg, Russia, Sept.27-28,2016. Atlantis Press, Electronic product, Paris, France, pp. 447-450.

[6] Yanmei Zhang and Han DING, Pronouns Study in China-Taking a Research on Pronouns in Excavated Materials of Pre-Qin Dynasty as an Example, 2016 3rd International Conference on Education, Language, Art and Inter-cultural Communication (ICELAIC 2016), Xiamen, Fujian, China, December 2-4, 2016. Atlantis Press, Electronic product, Paris, France, pp. 360-363. 